\title{
Quenching of Exciton Recombination in Strained Two-Dimensional Monochalcogenides
}

\author{
J. J. Esteve-Paredes, ${ }^{1, *}$ Sahar Pakdel, ${ }^{1,2,3, \dagger}$ and J. J. Palacios ${ }^{1,4,5,+}$ \\ ${ }^{1}$ Departamento de Física de la Materia Condensada, Universidad Autónoma de Madrid, E-28049 Madrid, Spain \\ ${ }^{2}$ Department of Physics and Astronomy, Aarhus University, 8000 Aarhus C, Denmark \\ ${ }^{3}$ School of Electrical and Computer Engineering, University College of Engineering, \\ University of Tehran, Tehran 14395-515, Iran \\ ${ }^{4}$ Instituto Nicolás Cabrera (INC) and Condensed Matter Physics Center (IFIMAC), \\ Universidad Autónoma de Madrid, E-28049 Madrid, Spain \\ ${ }^{5}$ Department of Physics, University of Texas, Austin, Texas 78712, USA
}

(Received 17 April 2019; published 14 August 2019)

\begin{abstract}
We predict that long-lived excitons with very large binding energies can also exist in a single or few layers of monochalcogenides such as GaSe. Our theoretical study shows that excitons confined by a radial local strain field are unable to recombine despite electrons and holes coexisting in space. The localized single-particle states are calculated in the envelope function approximation based on a three-band $\boldsymbol{k} \cdot \boldsymbol{p}$ Hamiltonian obtained from density-functional-theory calculations. The binding energy and the decay rate of the exciton ground state are computed after including correlations in the basis of electron-hole pairs. The interplay between the localized strain and the caldera-type valence band characteristic of few-layered monochalcogenides creates localized electron and hole states with very different quantum numbers which hinders the recombination even for singlet excitons.
\end{abstract}

DOI: 10.1103/PhysRevLett.123.077402

Introduction.-Long-lived excitons have been long sought after both for creating quantum Bose gases in semiconducting materials [1,2] and for the development of novel excitonic devices. To date, these have been extensively studied in III-V and II-VI semiconductor heterostructures where the range of existence is limited to low temperatures. Very recently, they have also been observed at room temperature in van der Waals transition-metal dichalcogenide (TMDC) two-dimensional (2D) heterostructures $[3,4]$. In both cases, the long-lived nature stems from the fact that the electron and the hole are spatially separated, thereof the name indirect excitons.

The spatial separation between electron and hole composing an indirect or interlayer exciton in TMDC 2D heterostructures allows for long recombination lifetimes, but it is also a limitation. Leaving aside the technical difficulties of creating heterostructures in a controlled manner, the required spatial separation limits, in turn, the binding energy. Larger interlayer or electronhole distances amount to longer recombination times but weaker binding energies. Here we show that both requirements, impossible to meet in TMDC heterostructures, can be found in strained few-layered monochalcogenides such as GaSe.

It is well known that the application of external strain on a semiconductor leads to a modification of the band gap. This is particularly important in 2D crystals where large strains can be applied before the crystal ruptures $[5,6]$. The modulation of the band gap due to strain has been quantified for $\mathrm{MoS}_{2}$ [7], phosphorene [8], and GaSe [9], to name a few. In the multilayer form of the latter and in other 2D TMDC, a causality relation between localized strain deformations and single-photon emission has been demonstrated [10-14], although the ultimate reason for this relation remains unclear.

The proven strong band-gap modulation with strain, the possibility of single-photon emission, along with an anomalous shape of the GaSe valence band motivates the present study. Below approximately seven layers, the valence band turns from a common inverted parabola to a caldera-type shape or ring-type shape $[9,15]$. Free excitons in GaSe monolayer have already been studied with ab initio methods [16], showing a variety of behaviors due to the peculiar valence band. Here we are interested in localized radial deformations as those invoked to explain single-photon emission in multilayer GaSe [10,11]. Here we show that the combined peculiarity of the valence band along with local strain quenches the photoluminescence by making the localized excitons extremely long-lived, as shown below.

The effective Hamiltonian.-We start by constructing an accurate low-energy Hamiltonian for a monolayer of GaSe using $\boldsymbol{k} \cdot \boldsymbol{p}$ theory. First, we find the density-functionaltheory (DFT) band structure of monolayer GaSe as shown in Fig. 1 (blue solid lines). We have used the CRYSTAL code implementation of the hybrid functional HSE06 [17] to obtain a good approximation of the actual quasiparticle gap. Our DFT calculation will serve a twofold purpose: to check the validity of our effective model Hamiltonian and to obtain the necessary information (Bloch states and band 


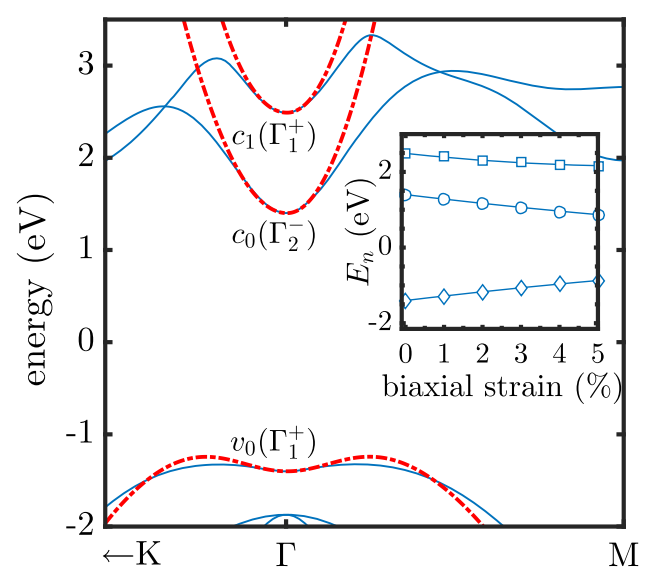

FIG. 1. DFT (solid blue lines) and effective bands (red dashed lines) of a GaSe monolayer along the $K-\Gamma-M$ path near the $\Gamma$ point. IRs of the zone center states are shown according to Ref. [18]. The inset shows the evolution of the energies at the $\Gamma$ point for several biaxial strain values calculated using DFT.

edges at the $\Gamma$ point) to compute the momentum matrix elements for a parameter-free description. The unperturbed Hamiltonian is $H_{n m}^{(0)}=E_{n} \delta_{n m}$, where the $n, m$ indices represent Bloch states at the $\Gamma$ point, which we will use as our basis, and $E_{n}$ is the energy of these Bloch states. Following the discussion in Ref. [18], we pursue a threeband model to properly account for the caldera form of the valence band (see Fig. 1).

In $\boldsymbol{k} \cdot \boldsymbol{p}$ theory, the first-order correction is linearly dependent in $k$ with the form $H_{n m}^{(1)}=\left(\hbar / m_{0}\right) \sum_{\alpha} k_{\alpha} p_{n m}^{\alpha}$, where $\alpha$ (and later $\beta$ ) denotes Cartesian coordinates, and $p_{n m}^{\alpha}$ is the $\alpha$ component of the momentum matrix element between $\Gamma$-point Bloch states. Based on group theory considerations, we can anticipate the momentum matrix elements that are nonzero and, thereby, the general form of the Hamiltonian. We will follow the notation in Ref. [18] for the $D_{3 h}$ point group to which monolayer GaSe belongs. The valence and the two first conduction bands $v_{0}, c_{0}$, and $c_{1}$ belong to the $\Gamma_{1}^{+}, \Gamma_{2}^{-}$, and $\Gamma_{1}^{+}$irreducible representations (IRs) respectively, and will form our working subspace $A$ from now on. In order for the momentum matrix elements to be nonzero, the decomposition of the direct product of the eigenstates IRs and the IR of the momentum operators, which in the $D_{3 h}$ group transform as $\Gamma_{3}^{+}\left(p_{x}, p_{y}\right)$ and $\Gamma_{2}^{-}$ $\left(p_{z}\right)$, must contain the fully symmetrical representation $\Gamma_{1}^{+}$. One can see that only $\left\langle\Gamma_{3}^{+}\left|p_{x, y}\right| \Gamma_{1}^{+}\right\rangle,\left\langle\Gamma_{3}^{+}\left|p_{x, y}\right| \Gamma_{2}^{+}\right\rangle$, $\left\langle\Gamma_{3}^{-}\left|p_{x, y}\right| \Gamma_{1}^{-}\right\rangle,\left\langle\Gamma_{3}^{-}\left|p_{x, y}\right| \Gamma_{2}^{-}\right\rangle,\left\langle\Gamma_{3}^{+}\left|p_{x, y}\right| \Gamma_{3}^{+}\right\rangle,\left\langle\Gamma_{3}^{-}\left|p_{x, y}\right| \Gamma_{3}^{-}\right\rangle$, and their complex conjugates are nonzero. Therefore, the resulting Hamiltonian will not contain terms linear in $k$. The absence of linear terms in the Hamiltonian forces us to consider the coupling of the subspace $A$ with the rest of the bands, which now becomes the dominant contribution. This is typically done through the Löwdin partitioning technique [19]. Including the coupling with all bands and evaluating numerically the momentum matrix elements, the effective Hamiltonian becomes

$$
H^{\mathrm{eff}}(\boldsymbol{k})=\left(\begin{array}{ccc}
A(\boldsymbol{k}) & 0 & B(\boldsymbol{k}) \\
0 & C(\boldsymbol{k}) & 0 \\
B^{*}(\boldsymbol{k}) & 0 & D(\boldsymbol{k})
\end{array}\right)
$$

with

$$
\begin{aligned}
& A(\boldsymbol{k})=-1.4+19.6 k_{x}^{2}+20.0 k_{y}^{2}, \\
& B(\boldsymbol{k})=55.5 k_{x}^{2}+63.4 k_{y}^{2}, \\
& C(\boldsymbol{k})=1.4+67.7 k_{x}^{2}+164.8 k_{y}^{2}, \\
& D(\boldsymbol{k})=2.5+74.3 k_{x}^{2}+148.8 k_{y}^{2}
\end{aligned}
$$

in units of $\mathrm{eV}$ (and atomic units for wave vectors). As discussed earlier, $v_{0}$ and $c_{1}$ bands transform as $\Gamma_{1}^{+}$, which only couples with $\Gamma_{3}^{+}$states. On the other hand, $\Gamma_{3}^{+}$ does not couple with $\Gamma_{2}^{-}$, the IR of $c_{0}$. As a result, one could have expected $v_{0}$ and $c_{1}$ to remain decoupled from $c_{0}$ when evaluating the second-order correction in the partitioning method, as can actually be seen in Eq. (1). The three bands resulting from the effective Hamiltonian are shown in Fig. 1 (red dashed lines). The agreement with the original DFT bands is very good near the $\Gamma$ point included the caldera shape of the valence band. Since we have used all the bands in the evaluation of the second-order perturbation terms, no further improvement can be envisioned to second order within our three-band model.

Local strain model.-Assuming that strain changes occur on length scales much larger than the lattice spacing, we can work in the envelope function framework where one writes the eigenfunctions in the form $\psi_{\gamma}=\sum_{n} F_{n}^{(\gamma)}(\boldsymbol{r}) u_{n \mathbf{0}}(\boldsymbol{r})$. Here, $u_{n \mathbf{0}}$ are the periodic Bloch functions at $\boldsymbol{k}=\mathbf{0}$, and $F_{n}^{(\gamma)}$ are 2D envelope functions [20] calculated from a set of coupled differential equations:

$$
\sum_{m} H_{n m}^{\mathrm{SQD}}\left(\boldsymbol{r},-i \nabla_{\boldsymbol{r}}\right) F_{m}^{(\gamma)}(\boldsymbol{r})=\varepsilon_{\gamma} F_{n}^{(\gamma)}(\boldsymbol{r}) .
$$

$H^{\mathrm{SQD}}$ is the Hamiltonian of the strain-induced quantum dot (SQD) created by the local perturbation. We assume the strain to be locally biaxial, which can be modeled by adding a local "potential" to the $k$-independent diagonal terms in $H^{\text {eff }}$,

$$
H_{n n}^{\mathrm{SQD}}=E_{n}+\Delta_{n}(\boldsymbol{r})+\sum_{\alpha} M_{n}^{\alpha} \nabla_{\alpha}^{2}
$$

while keeping unchanged the $k$-dependent diagonal (here generically represented by $M$ ) and nondiagonal terms $B^{(*)}(\boldsymbol{k})$. This approximation captures the main effect, which is the confinement of electrons and holes, while maintaining the complexity of the implementation to a minimum. It neglects changes in the effective mass or band curvature and the possible appearance of new Hamiltonian terms due to the breaking of the $C_{3 h}$ symmetry, but it has been shown to be 


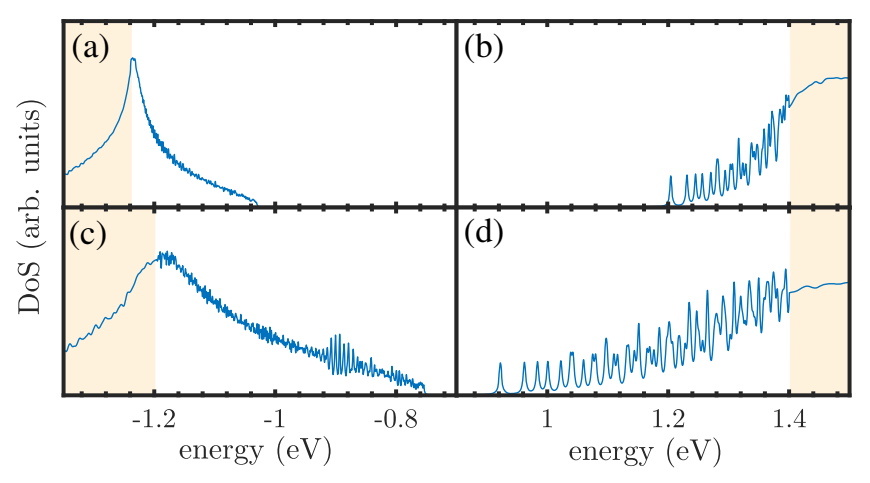

FIG. 2. Density of states of the valence band calculated from the numerical diagonalization of a SQD with $\sigma_{s}=10 \mathrm{~nm}, S_{0}=$ $2 \%$ (a) and $\sigma_{s}=10 \mathrm{~nm}, S_{0}=5 \%$ (c). Same for the conduction band [(b) and (d)]. We have used $3600 \mathrm{HO}$ basis functions for every envelope function in the two calculations with a $\mathrm{HO}$ frequency optimal to match the Gaussian curvature of the strain field. Extended states approximately belong in the shaded regions.

quantitatively accurate in a somewhat similar context [21]. In practice, we consider a tensile Gaussian strain field of the form $S_{0} \exp \left(-r^{2} / \sigma_{s}^{2}\right)$ with a maximum strain at its center of $5 \%$. The local lattice deformation is mapped onto $\Delta_{n}(r)$ thanks to a series of DFT calculations performed with biaxial tensile strain. In the inset of Fig. 1, we show the $\Gamma$-point energies as obtained for several values of the tensile strain. We have also implicitly assumed, as seen in the inset of Fig. 1, that the chemical potential (set at zero) stays in the midgap at all points in space. A detailed calculation is beyond the scope of this work, but this choice complies with local charge neutrality and overall thermodynamic equilibrium at low temperatures.

Single-particle results. - In order to solve Eq. (3), we further expand the envelope functions as

$$
F_{n}^{(\gamma)}=\sum_{n_{x} n_{y}} c_{n_{x} n_{y}}^{(n \gamma)} \phi_{n_{x} n_{y}}
$$

where $\phi_{n_{x} n_{y}}$ are the Cartesian 2D harmonic oscillator (HO) basis functions. The kinetic part of the matrix elements in the $\mathrm{HO}$ basis can be easily evaluated using the ladder operators, while the strain contribution coming from the term $\Delta_{n}(r)$ is evaluated numerically. As an example, we show in Fig. 2 the density of states of electrons and holes obtained from the numerical diagonalization of Eq. (3) for two representative cases. In both cases, we find bound states within the band gap of the unstrained monolayer. These states distribute in a more dense spectrum in the valence band than in the conduction band, a consequence of the caldera shape of the former, which also gives rise to the expected van Hove singularity [16]. The number of bound states in each band increases with $\sigma_{s}$ and $S_{0}$, as could intuitively be expected. The transition from bound to delocalized states (shaded regions in Fig. 2) occurs near
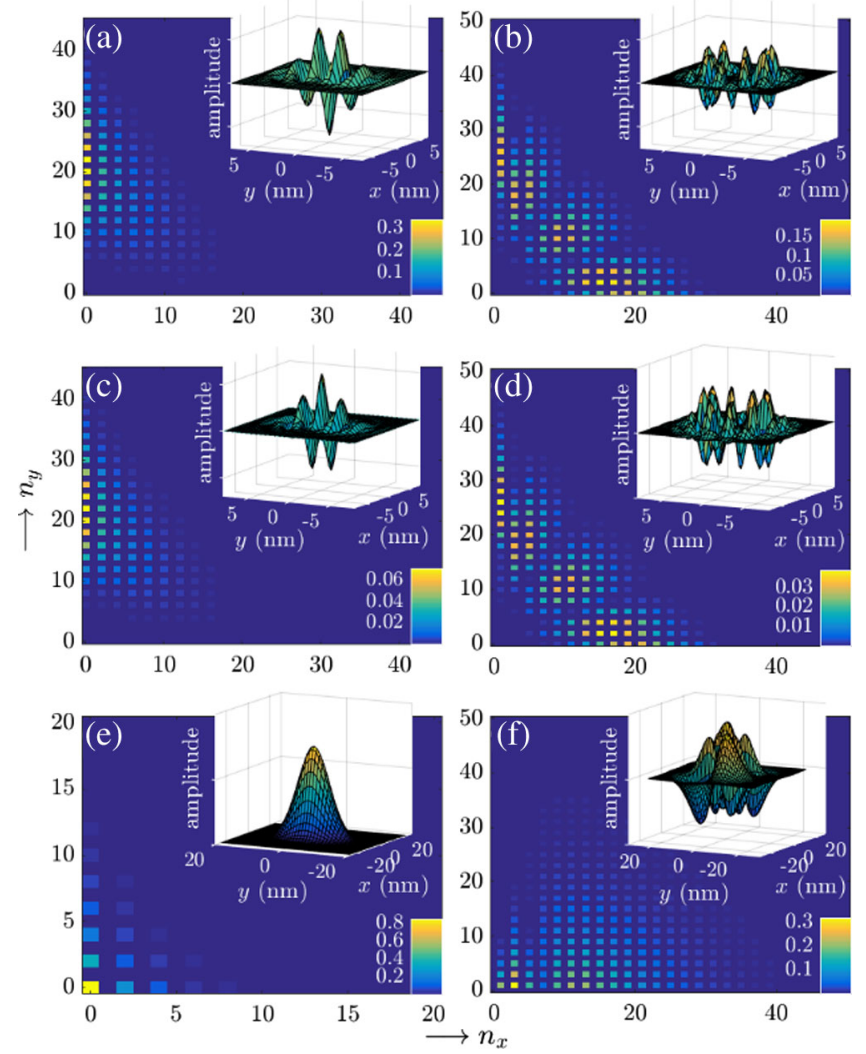

FIG. 3. Charts of the HO expansion (absolute value of the coefficients) for the envelope functions. $v_{0}$ envelope function for (a) the first and (b) the 20th valence state. (c) and (d) panels show the $c_{1}$ envelope function for the same states (notice the much smaller contribution). $c_{0}$ envelope function for (e) the first and (f) the 20th conduction state. The insets show the actual shape of the associated wave function in real space.

the band edges of the unstrained conduction and valence bands. A minimum of $\sim 30$ and $\sim 100$ bound states have been found in the valence and conduction band, respectively, in all the studied cases, which is sufficient for our purposes.

A plot of the envelope functions reveals the very different nature of valence and conduction bound states. In Figs. 3(a) and 3(c), we show a chart of the HO basis coefficients (absolute value) of the $v_{0}$ and $c_{1}$ envelope functions corresponding to the highest-energy hole state and in Fig. 3(e) the $c_{1}$ envelope function of the lowestenergy electron state. Both hole envelope functions show a very similar behavior, although $v_{0}$ gives the main contribution to the state. The other envelope function is zero due to the absence of coupling in Eq. (1). The electron state has mainly $\left(n_{x}=0, n_{y}=0\right)$ character (as could be anticipated for a parabolic band), but the hole state shows a dramatic shift to high $n_{y}$ quantum numbers. This behavior can be traced back to the caldera ring away from the $\Gamma$ point in the unstrained band structure. We also show the 20th electron and hole states (moving away from the band edges) in Figs. 3(b), 3(d), and 3(f). The hole state spreads now also to 
higher values of $n_{x}$, while the electron state still presents the largest weight at low $\mathrm{HO}$ quantum numbers.

Excitons.-Interactions are now added to the singleparticle Hamiltonian expanded by electron and hole bound states:

$$
H=H^{\mathrm{SQD}}+H^{e, h},
$$

with

$$
\begin{aligned}
H^{\mathrm{SQD}} & =\sum_{i} \epsilon_{i} c_{i}^{\dagger} c_{i}+\sum_{j} \epsilon_{j} d_{j}^{\dagger} d_{j}, \\
H^{e, h} & =-\sum_{\substack{i_{1}, j_{1}, i_{2}, j_{2} \\
\sigma, \sigma^{\prime}}} c_{i_{1} \sigma}^{\dagger} c_{i_{2} \sigma} d_{j_{2}\left(-\sigma^{\prime}\right)}^{\dagger} d_{j_{1}\left(-\sigma^{\prime}\right)} V_{i_{1}, j_{1}, j_{2}, i_{2}} .
\end{aligned}
$$

In the electron-hole interaction terms, we omit the exchange part as, in the leading order, their matrix elements would give no energy corrections $[22,23]$. This is due to the specific form of our Hamiltonian in Eq. (1), which results in conduction and valence states not sharing envelope functions.

No other interaction terms are needed since we only consider single electron-hole pairs. At this level, excitonic states can be constructed as

$$
|X\rangle=\sum_{i, j, \sigma} A_{i j} c_{i \sigma}^{\dagger} d_{j(-\sigma)}^{\dagger}|0\rangle
$$

where $|0\rangle$ denotes the vacuum state with no electrons and holes.

The excitonic states are then calculated by projecting Eq. (7) onto the electron-hole pair basis and converged in the number of these pairs (we restrict ourselves to 200 electron-hole pairs which is enough for our purposes).

For the interaction kernel, we have considered the bare Coulomb interaction and the Keldysh interaction model, more appropriate for 2D systems [24]. Both types of interactions give qualitatively similar results with unimportant differences in the actual exciton binding energies which are not relevant for our discussion here. Since the interactions preserve all the symmetries, excitons can be classified according to how the transform under in-plane reflections in both electron and hole coordinates. In the following, we focus on the excitons whose electron and hole wave functions transform similarly, as these are, in principle, the active ones in photoluminescence.

In Fig. 4(a), we show the quasiparticle gap and binding energy of the optically active (symmetry-allowed) lowestenergy excitonic state $E_{X}^{(0)}$ for different parameters characterizing our SQD. Depending on the parameters and interaction details, this can be the ground state, but this is unimportant for our main conclusion (see below). In particular, we have used the Keldysh potential [25] in an environment of vacuum. The binding energies decrease with $\sigma_{s}$ since the single-particle states become more extended in space. On the other hand, the binding energy

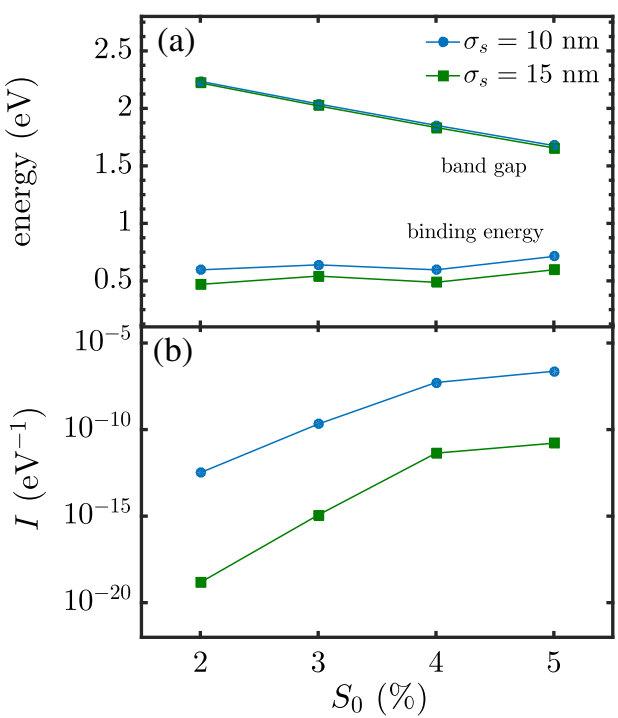

FIG. 4. (a) Band gap and binding energy of the ground state exciton for several values of the strain strength and extension. The Keldysh interaction with a screening length $r_{0}=10 \AA$ has been used. (b) Decay rate of the ground state exciton as expressed in Eq. (12) for the cases in (a).

increases with the strength of the strain as they become more localized.

Quenching of the exciton recombination.-The most relevant result is found in the photoluminescence. The recombination rate to the ground state mediated by the emission of a photon can be computed using Fermi's golden rule (ignoring proportionality constants) [23,26,27]

$$
I \propto \frac{1}{E_{X}^{(0)}} \sum_{\boldsymbol{q} \lambda}\left|\sum_{i, j} A_{i j} p_{j i}^{(\boldsymbol{q} \lambda)}\right|^{2} \delta\left(E_{X}^{(0)}-\hbar \omega_{q}\right) .
$$

Here, $p_{j i}^{(q \lambda)}$ is the scalar product of the momentum matrix element and the photon polarization vector $\boldsymbol{\epsilon}_{\boldsymbol{q} \lambda}$. The squared quantity is known as the oscillator strength. Thus, the recombination is encoded in those electron-hole pairs which have a relevant contribution to the exciton wave function. The momentum matrix element in Eq. (9) reads

$$
p_{j i}^{(\boldsymbol{q} \lambda)}=\sum_{m, n \in A}\left\langle u_{m}\left|\boldsymbol{\epsilon}_{\boldsymbol{q} \lambda} \cdot \boldsymbol{p}\right| u_{n}\right\rangle\left\langle F_{m}^{(j)} \mid F_{n}^{(i)}\right\rangle .
$$

Additional terms would appear if one considers the firstorder correction $\psi_{\gamma}^{(1)}=\sum_{m \notin A} \tilde{F}_{m}^{(\gamma)} u_{m 0}$ to the bound states, with the $\tilde{F}_{m}$ being envelope functions obtained in terms of the zero-order ones. As explained below, these corrections can be safely neglected.

For in-plane polarization, we have already seen that the momentum matrix element between states in $A$ vanish. If the polarization vector has a $z$ component, then the only coupling allowed is between $v_{0}$ and $c_{0}$ [16]. Thus, in the leading order 


$$
p_{j i}^{(\boldsymbol{q} \lambda)} \approx\left\langle u_{v_{0}}\left|p_{z} \cos \theta_{z}\right| u_{c_{0}}\right\rangle\left\langle F_{v_{0}}^{(j)} \mid F_{c_{0}}^{(i)}\right\rangle,
$$

being $\cos \theta_{z}$ the $z$-direction cosine of the polarization vector. For a single polarization, Eq. (9) reduces to

$$
I \propto \frac{\left|\sum_{i j}\left\langle F_{v_{0}}^{(j)} \mid F_{c_{0}}^{(i)}\right\rangle\right|^{2}}{E_{X}^{(0)}} \delta\left(E_{X}^{(0)}-\hbar \omega_{q}\right) .
$$

Since we are considering symmetry-allowed active excitons, the scalar product can, in principle, be nonzero. Common semiconductors present parabolic bands for both electrons and holes and an effective mass in each band can be defined. When confined, their envelope wave functions thus present a very similar quantum character (similar quantum numbers when expressed in some basis) and a large overlap. Here, electrons have indeed an effective mass, but this quantity is ill-defined for the valence band. While the relevant electron wave functions are smooth and present no or few nodes (see Fig. 3), the relevant valence wave functions present many nodes since, ultimately, they originate from different Bloch states away from the center of the Brillouin zone. Therefore, we can conjecture that even the dipole-allowed transitions will vanish due to the nearly orthogonal nature of the hole and electron envelope functions.

In Fig. 4(b) we show the rate of Eq. (12) in logarithmic scale. We obtain, as expected, very low values for all the cases studied (in contrast to values of the order of unity in the case of the most common semiconductors). Higherorder corrections to the envelope functions $\psi_{\gamma}^{(1)}$ are not expected to give a relevant contribution to Eq. (10). These are obtained by differentiation of the zero-order envelope functions [19], which translates in a spreading of the order of unity of the quantum numbers (see, e.g., Fig. 3), thus maintaining its quasiorthogonal character. Finally, note that this result does not depend on the singlet or triplet nature of the exciton or the magnitude of the dipolar matrix elements because it is encoded in the envelope part of the wave functions. We thus conclude that photoemission is generically quenched.

Conclusions and perspectives.-We have shown that excitons localized by a radial strain perturbation in GaSe monolayer present extremely long recombination times. This is a direct consequence of the very different quantum nature of the confined wave function of electrons and holes. Although we have focused on a specific 2D monochalcogenide, everything seems to indicate that similar results would be obtained in other monochalcogenides such as $\mathrm{GaS}$ and InSe [15] and even in different families of materials presenting a caldera-shaped valence or conduction band. The study of many-body effects beyond singleexciton physics also presents intriguing possibilities in these materials which will be explored in the future.

Research supported by the Spanish MINECO through Grant No. FIS2016-80434-P and the María de Maeztu
Programme for Units of Excellence in Research and Development (MDM-2014-0377), the Fundación Ramón Areces, and the European Union Seventh Framework Programme under Grant Agreement No. 604391 Graphene Flagship. S.P. was also supported by the VILLUM FONDEN via the Centre of Excellence for Dirac Materials (Grant No. 11744). We acknowledge the computer resources and assistance provided by the Centro de Computación Científica of the Universidad Autónoma de Madrid.

*juan.esteve@uam.es pakdel.sahar@phys.au.dk *juanjose.palacios@uam.es

[1] Y. E. Lozovik and V. I. Yudson, Zh. Eksp. Teor. Fiz. 71, 738 (1976) [Sov. Phys. JETP 44, 389 (1976)].

[2] L. V. Butov, A. L. Ivanov, A. Imamoglu, P. B. Littlewood, A. A. Shashkin, V. T. Dolgopolov, K. L. Campman, and A. C. Gossard, Phys. Rev. Lett. 86, 5608 (2001).

[3] D. Unuchek, A. Ciarrocchi, A. Avsar, K. Watanabe, T. Taniguchi, and A. Kis, Nature (London) 560, 340 (2018).

[4] E. Calman, M. Fogler, L. Butov, S. Hu, A. Mishchenko, and A. Geim, Nat. Commun. 9, 1895 (2018).

[5] C. Lee, X. Wei, J. W. Kysar, and J. Hone, Science 321, 385 (2008).

[6] S. Bertolazzi, J. Brivio, and A. Kis, ACS Nano 5, 9703 (2011).

[7] A. Castellanos-Gomez, R. Roldn, E. Cappelluti, M. Buscema, F. Guinea, H. S. J. van der Zant, and G. A. Steele, Nano Lett. 13, 5361 (2013).

[8] J. Quereda, P. San-Jose, V. Parente, L. Vaquero-Garzon, A. J. Molina-Mendoza, N. Agraït, G. Rubio-Bollinger, F. Guinea, R. Roldán, and A. Castellanos-Gomez, Nano Lett. 16, 2931 (2016).

[9] D. Maeso, S. Pakdel, H. Santos, N. Agrait, J. J. Palacios, E. Prada, and G. Rubio-Bollinger, Nanotechnology 30 24LT01 (2019).

[10] J. Kern, I. Niehues, P. Tonndorf, R. Schmidt, D. Wigger, R. Schneider, T. Stiehm, S. Michaelis de Vasconcellos, D. E. Reiter, T. Kuhn et al., Adv. Mater. 28, 7101 (2016).

[11] P. Tonndorf, S. Schwarz, J. Kern, I. Niehues, O. D. Pozo-Zamudio, A. I. Dmitriev, A. P. Bakhtinov, D. N. Borisenko, N. N. Kolesnikov, A. I. Tartakovskii et al., 2D Mater. 4, 021010 (2017).

[12] P. Tonndorf, R. Schmidt, R. Schneider, J. Kern, M. Buscema, G. A. Steele, A. Castellanos-Gomez, H. S. J. van der Zant, S. M. de Vasconcellos, and R. Bratschitsch, Optica 2, 347 (2015).

[13] S. Kumar, A. Kaczmarczyk, and B. D. Gerardot, Nano Lett. 15, 7567 (2015).

[14] P. Tonndorf, O. Del Pozo-Zamudio, N. Gruhler, J. Kern, R. Schmidt, A. I. Dmitriev, A. P. Bakhtinov, A. I. Tartakovskii, W. Pernice, S. Michaelis de Vasconcellos et al., Nano Lett. 17, 5446 (2017).

[15] D. V. Rybkovskiy, A. V. Osadchy, and E. D. Obraztsova, Phys. Rev. B 90, 235302 (2014).

[16] G. Antonius, D. Y. Qiu, and S. G. Louie, Nano Lett. 18, 1925 (2018). 
[17] R. Dovesi, R. Orlando, A. Erba, C. M. Zicovich-Wilson, B. Civalleri, S. Casassa, L. Maschio, M. Ferrabone, M. De La Pierre, P. D'Arco et al., Int. J. Quantum Chem. 114, 1287 (2014).

[18] P. Li and I. Appelbaum, Phys. Rev. B 92, 195129 (2015).

[19] S. Glutsch, Excitons in Low-Dimensional Semiconductors: Theory Numerical Methods Applications, Springer Series in Solid-State Sciences (Springer, Berlin, 2004).

[20] Note that in the expansion of the SQD states, the envelope functions are defined in 2D while the Bloch periodic functions are $a b$ initio 3D functions.
[21] M. Tadić, F. M. Peeters, K. L. Janssens, M. Korkusiski, and P. Hawrylak, J. Appl. Phys. 92, 5819 (2002).

[22] M. Azizi and P. Machnikowski, Phys. Rev. B 91, 195314 (2015).

[23] W. Sheng, S.-J. Cheng, and P. Hawrylak, Phys. Rev. B 71, 035316 (2005).

[24] L. V. Keldysh, Sov. Phys. JETP 29, 658 (1979).

[25] E. Prada, J. V. Alvarez, K. L. Narasimha-Acharya, F. J. Bailen, and J. J. Palacios, Phys. Rev. B 91, 245421 (2015).

[26] P. Hawrylak, Phys. Rev. B 60, 5597 (1999).

[27] C. D. Spataru, S. Ismail-Beigi, R. B. Capaz, and S. G. Louie, Phys. Rev. Lett. 95, 247402 (2005). 